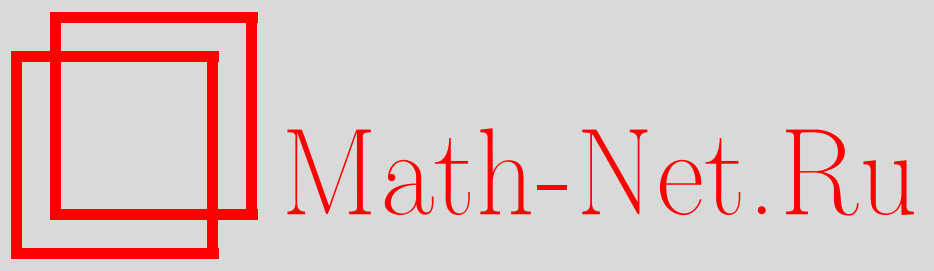

А. М. Зубков, А. А. Серов, Совокупность образов подмножества конечного множества при итерациях случайных отображений, Дискрет. матем., 2014, том 26, выпуск 4, 43-50

DOI: https://doi.org/10.4213/dm1303

Использование Общероссийского математического портала Math-Net.Ru подразумевает, что вы прочитали и согласны с пользовательским соглашением http: //www . mathnet.ru/rus/agreement

Параметры загрузки:

IP : 54.197 .217 .227

26 апреля 2023 г., 15:07:21

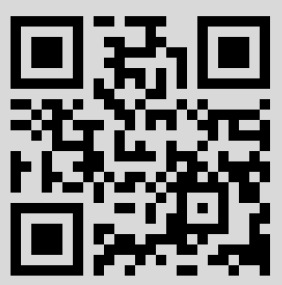




\title{
Совокупность образов подмножества конечного множества при итерациях случайных отображений
}

\author{
() 2014 г. А. М. Зубков* , А. А. Серов**
}

Пусть $\mathcal{N}$ - множество из $N$ элементов и $F_{1}, F_{2}, \ldots$ - последовательность случайных независимых равновероятных отображений $\mathcal{N} \rightarrow \mathcal{N}$. Для подмножества $S_{0} \subset \mathcal{N},\left|S_{0}\right|=n$, рассматриваются последовательность его образов $S_{k}=F_{k}\left(\ldots F_{2}\left(F_{1}\left(S_{0}\right)\right) \ldots\right), k=1,2 \ldots$, и последовательность их объединений $\Psi_{k}=S_{1} \cup \ldots \cup S_{k}, k=1,2 \ldots$ Описан способ точного вычисления распределений $\left|S_{k}\right|$ и $\left|\Psi_{k}\right|$ при умеренных значениях $N$. Получены двусторонние неравенства для $\mathbf{M}\left|S_{k}\right|$ и $\mathbf{M}\left|\Psi_{k}\right|$, в которых верхние оценки асимптотически эквивалентны нижним, если $N, n, k \rightarrow \infty, n k=o(N)$. Результаты представляют интерес для анализа алгоритмов балансировки времени и памяти.

Ключевые слова: итерации случайных отображений, метод балансировки времени и памяти.

\section{1. Введение}

Одной из известных вычислительно труднорешаемых задач является поиск решения уравнения

$$
G(x)=a,
$$

где $G$ - такое отображение конечного множества $\mathcal{N}=\{1, \ldots, N\}$ в себя, что все известные способы вычисления значения $G^{-1}(a)$ по трудоемкости сравнимы с перебором всего множества $\mathcal{N}$. Очевидным методом поиска решения уравнения (1) является последовательное вычисление значений $G(x)$ для всех $x \in \mathcal{N}$ до тех пор, пока не обнаружится решение этого уравнения. Для реализации такого метода требуется память медленно растущего при $N \rightarrow \infty$ объема (необходимого для вычисления значений функции $G$ при любом $x \in \mathcal{N}$ ), но время работы (число операций) при использовании этого метода имеет порядок $O(N)$.

M. E. Hellman [10] предложил универсальный (не зависящий от вида функции $G$ ) метод поиска решений уравнения (1), позволяющий (после предварительного этапа, проводимого за время порядка $O(N))$ за счет использования памяти (по порядку меньшей $O(N))$ находить решение каждого уравнения (1) с большой вероятностью за время, по порядку меньшее $O(N)$. Этот подход был назван балансировкой

* Место работы: Математический институт им. В.А. Стеклова РАН, e-mail: zubkov@mi.ras.ru

** Место работы: Математический институт им. В.А. Стеклова РАН, e-mail:serov@mi.ras.ru 
времени и памяти. На предварительном этапе метода Хеллмана и его более поздних модификаций с помощью вычисления $n t^{2}=O(N)$ значений суперпозиций вида $F=R(G(x))$ (где отображения $R: \mathcal{N} \rightarrow \mathcal{N}$ выбираются тем или иным способом) составляются таблицы, содержащие в совокупности $O(N / t)$ пар вида $\left(x, F^{t}(x)\right)$, где $F^{t}(x)-t$-кратная итерация отображений вида $F=R(G(x))$; эти таблицы позволяют на основном этапе для любого $a \in \mathcal{N}$ находить решение уравнения (1) с помощью вычисления $O(N / n)$ значений вида $R(G(x))$. Если $n$ и $t$ имеют порядок $O\left(N^{1 / 3}\right)$, то объем таблиц, составленных на предварительном этапе, имеет порядок $O\left(N^{2 / 3}\right)$, а на основном этапе нахождение каждого решения имеет сложность порядка $O\left(N^{2 / 3}\right)$ вычислений значений $R(G(x))$ (все эти оценки приведены с точностью до логарифмических множителей).

Мы рассматриваем упрощенную математическую модель процесса построения одной «радужной» таблицы (эта модель соответствует варианту метода балансировки времени и памяти, предложенному в [15]). Модель имеет следующий вид: в множестве $\mathcal{N}$ выбирается начальное подмножество $S_{0},\left|S_{0}\right|=n$, и вычисляются его образы

$$
S_{1}=F_{1}\left(S_{0}\right), S_{2}=F_{2}\left(F_{1}\left(S_{0}\right)\right), \ldots, S_{t}=F_{t}\left(F_{t-1}\left(\ldots\left(F_{1}\left(S_{0}\right)\right) \ldots\right)\right),
$$

где $F_{1}, \ldots, F_{t}$ - независимые случайные отображения множества $\mathcal{N}$ в себя, имеющие равновероятное распределение на множестве $\Sigma_{N},\left|\Sigma_{N}\right|=N^{N}$, всех таких отображений.

В статье описан способ точного вычисления распределений случайных величин $\varphi_{k}=\left|S_{k}\right|$ и $\zeta_{t}=\left|S_{1} \cup S_{2} \cup \ldots \cup S_{t}\right|$ с помощью цепей Маркова, применимый при умеренных значениях $N$, и получены двусторонние оценки математических ожиданий этих случайных величин и вероятностей того, что элемент $x \in \mathcal{N}$, не зависящий от итерируемых отображений $F_{1}, F_{2}, \ldots$, принадлежит множеству $S_{k}$ или множеству $S_{1} \cup S_{2} \cup \ldots \cup S_{t}$. Верхние и нижние оценки асимптотически эквивалентны при $N, n, t \rightarrow \infty$, если $n t=o(N)$. Эти результаты могут использоваться для оптимизации методов балансировки времени и памяти.

Характеристики методов балансировки времени и памяти, а также свойств итераций случайных отображений изучались в ряде работ. Перечислим некоторые полученные в них результаты.

В [10] для случайного равновероятного отображения $F: \mathcal{N} \rightarrow \mathcal{N}$, множества $S \subset$ $\mathcal{N}$ мощности $n$ : $|S|=n$, и случайного множества $\Phi_{t}=F(S) \cup F^{2}(S) \cup \ldots \cup F^{t}(S)$, где $F^{k}(S)$ - образ $S$ при $k$-кратной итерации отображения $F, k=1,2, \ldots$, получены верхняя и нижняя оценки вероятности того, что $x \in \Phi_{t}$ для любого $x \in \mathcal{N}$, а именно:

$$
\frac{1}{N} \sum_{i=1}^{n} \sum_{j=1}^{t}\left(1-\frac{i t}{N}\right)^{j} \leqslant \mathbf{P}\left\{x \in \Phi_{t}\right\} \leqslant \frac{n t}{N} .
$$

В [10] показано, что при $n t^{2} \approx N$ и $n, t \gg 1$ левая часть этого неравенства близка к $0.80 \frac{n t}{N} ;$ в [13] получена оценка

$$
\frac{1}{N} \sum_{i=1}^{n} \sum_{j=1}^{t}\left(1-\frac{i t}{N}\right)^{j} \geqslant \frac{n t}{N} \int_{0}^{1} \frac{1-e^{-x}}{x} d x \approx 0.796599 \frac{n t}{N} .
$$

Приведенное в [10] доказательство неравенства (2) справедливо и для итераций независимых случайных отображений. 
В [11] с помощью перехода к аппроксимирующим дифференциальным уравнениям получена другая приближенная формула:

$$
\mathbf{P}\left\{x \in \Phi_{t}\right\} \approx 2\left(1-\frac{1}{\tau^{2}}\right) \frac{e^{t / \tau}-e^{-t / \tau}}{(\tau+1) e^{t / \tau}+(\tau-1) e^{-t / \tau}},
$$

где $\tau=\sqrt{\frac{2 N}{n}}$.

В [15] для случайного равновероятного отображения $F: \mathcal{N} \rightarrow \mathcal{N}$, последовательности $R_{1}, R_{2}, \ldots$ случайных независимых взаимно однозначных отображений $\mathcal{N} \rightarrow \mathcal{N}$, подмножества $S \subset \mathcal{N},|S|=n$, последовательности его образов $S_{k}=R_{k}\left(F\left(\ldots R_{2}\left(F\left(R_{1}(F(S))\right)\right) \ldots\right)\right), k=1,2, \ldots$, и случайного множества $\Psi_{k}=S_{1} \cup \ldots \cup S_{k}, k=1,2, \ldots$, с использованием эвристических рассуждений получена приближенная формула

$$
\mathbf{P}\left\{x \in S \cup \Psi_{t}\right\} \approx 1-\prod_{i=1}^{t}\left(1-\frac{n_{i}}{N}\right),
$$

где $n_{1}=n, n_{i+1}=N\left(1-e^{-\frac{n_{i}}{N}}\right)$ при $i \geqslant 1$.

В [16] предлагается для оценивания характеристик методов балансировки времени и памяти использовать в качестве математической модели ветвящиеся процессы Гальтона-Ватсона.

В [12] в качестве одной из моделей популяционной генетики рассматривались последовательность $F_{1}, F_{2}, \ldots$ независимых равновероятных отображений и случайная величина

$$
\tau_{N}=\min \left\{t:\left|F_{t}\left(F_{t-1}\left(\ldots F_{1}(\mathcal{N}) \ldots\right)\right)\right|=1\right\}
$$

- минимальное число итераций случайных отображений $\mathcal{N}$ в себя, при котором образом $\mathcal{N}$ оказывается одноэлементное множество. В [12] отмечено, что в этой модели при $N \rightarrow \infty$ распределения случайных величин $\zeta=\frac{1}{N} \tau_{N}$ сходятся к распределению суммы $\xi=\sum_{j=1}^{\infty} \xi_{j}$, где случайные величины $\xi_{1}, \xi_{2}, \ldots$ независимы и

$$
\mathbf{P}\left\{\xi_{j} \leqslant x\right\}=1-e^{-x j(j+1) / 2}, \quad x \geqslant 0, \quad j=1,2, \ldots
$$

Так как $\mathbf{E} \xi_{j}=2 /(j(j+1))$, то $\xi$ имеет конечное математическое ожидание:

$$
\mathbf{E} \xi=\sum_{j=1}^{\infty} \frac{2}{j(j+1)}=2 .
$$

Позднее эти утверждения доказывались разными способами (см., например, [6], [2], [8]). В [14] получено обобщение этих утверждений на итерации неравновероятных отображений (когда образы разных элементов независимы и имеют одно и то же распределение на $\mathcal{N})$.

\section{2. Основные результаты}

Пусть, как и ранее, $F_{1}, F_{2}, \ldots$ - независимые случайные отображения множества $\mathcal{N}=\{1, \ldots, N\}$ в себя, $S_{0} \subset \mathcal{N},\left|S_{0}\right|=n, S_{k}=F_{k}\left(S_{k-1}\right), \Psi_{k}=\cup_{j=1}^{k} S_{j}, k \geqslant 1$. Положим $\varphi_{0}=\left|S_{0}\right|, \zeta_{0}=0, \varphi_{k}=\left|S_{k}\right|, \zeta_{k}=\left|\Psi_{k}\right|, k \geqslant 1$. Так как отображения $F_{1}, F_{2}, \ldots$ независимы и одинаково распределены, то последовательности $\left\{\varphi_{k}\right\}_{k \geqslant 0}$ и $\left\{\zeta_{k}\right\}_{k \geqslant 0}$ являются цепями Маркова. 
Утверждение 1. Матрица переходных вероятностей цепи Маркова $\left\{\varphi_{k}\right\}_{k \geqslant 0}$ имеem вид

$$
\begin{gathered}
P=\left\|p_{i, j}\right\|_{i, j=1}^{N}, \\
p_{i, j}= \begin{cases}\left(\begin{array}{c}
N \\
j
\end{array}\right)\left(\frac{j}{N}\right)^{i} \sum_{m=0}^{j}(-1)^{m}\left(\begin{array}{c}
j \\
m
\end{array}\right)\left(1-\frac{m}{j}\right)^{i}, & 1 \leqslant j \leqslant i \leqslant N, \\
0, & j>i .\end{cases}
\end{gathered}
$$

Матрища переходных вероятностей иепи Маркова $\left\{\left(\varphi_{k}, \zeta_{k}\right)\right\}_{k \geqslant 0}$ имеет вид

$$
\begin{gathered}
Q=\left\|q_{(i, r),(j, s)}\right\|_{i, j, r, s=1}^{N}, \\
q_{(i, r),(j, s)}=\left\{\begin{array}{c}
p_{i, j} \frac{\left(\begin{array}{c}
N-r \\
s-r
\end{array}\right)\left(\begin{array}{c}
r \\
j-s+r
\end{array}\right)}{\left(\begin{array}{c}
N \\
j
\end{array}\right)}=\left(\begin{array}{c}
N-r \\
s-r
\end{array}\right)\left(\begin{array}{c}
r \\
j-s+r
\end{array}\right)\left(\frac{j}{N}\right)^{i} \sum_{m=0}^{j}(-1)^{m}\left(\begin{array}{c}
j \\
m
\end{array}\right)\left(1-\frac{m}{j}\right)^{i}, \\
\text { если } 1 \leqslant j \leqslant i \leqslant N, 1 \leqslant r \leqslant s \leqslant \min \{N, r+j\}, \\
\text { в остальных случаях. }
\end{array}\right.
\end{gathered}
$$

Доказательство. Согласно описанию модели вероятность перехода цепи Маркова $\left\{\varphi_{k}\right\}_{k \geqslant 0}$ за 1 шаг из состояния $i$ в состояние $j$ равна вероятности того, что при независимом равновероятном размещении $i$ частиц по $N$ ячейкам число занятых ячеек равно $j$ или, что то же самое, число пустых ячеек равно $N-j$. Формула для $p_{i, j}$ в формулировке утверждения совпадает с формулами $(1),(2)$ в [4], гл. $1, \S 1$.

Переход цепи $\left(\varphi_{k}, \zeta_{k}\right)=\left(\left|S_{k}\right|,\left|\Psi_{k}\right|\right)$ из состояния $(i, r)$ в состояния $(j, s)$ с помощью отображения $F_{k+1}$ можно разбить на два этапа: на первом этапе происходит переход от множества $S_{k},\left|S_{k}\right|=i$, к множеству $S_{k+1}$ с $\left|S_{k+1}\right|=j$ (этот переход не зависит от $\Psi_{k}$ и имеет такую же вероятность $p_{i, j}$, как в цепи Маркова $\left.\left\{\varphi_{k}\right\}\right)$, а на втором этапе строится множество $\Psi_{k+1}=\Psi_{k} \cup S_{k+1}$; при этом в силу равновероятности отображения $F_{k+1}$ и его независимости от $F_{1}, \ldots, F_{k}$ множество $S_{k+1}$ с $\left|S_{k+1}\right|=j$ имеет равновероятное распределение на совокупности всех $j$-элементных подмножеств множества $\mathcal{N}$; поэтому

$$
\mathbf{P}\left\{\left|\Psi_{k+1}\right|=s|| \Psi_{k}|=r,| S_{k+1} \mid=j\right\}=\frac{\left(\begin{array}{c}
N-r \\
s-r
\end{array}\right)\left(\begin{array}{c}
r \\
j-s+r
\end{array}\right)}{\left(\begin{array}{c}
N \\
j
\end{array}\right)} .
$$

Утверждение доказано.

Вероятности переходов цепи Маркова $\left\{\varphi_{k}\right\}_{k \geqslant 0}$ за $k$ шагов образуют матрицу $P^{(k)}=\left\|p_{(i, j)}^{(k)}\right\|_{i, j=1}^{N}=P^{k}$. Таким образом, наборы чисел $\left\{p_{(n, j)}^{(k)}=\mathbf{P}\left\{\varphi_{k}=j \mid \varphi_{0}=\right.\right.$ $n\}, j=1, \ldots, N\}$ задают распределения $\varphi_{k}$, что позволяет находить численные значения характеристик распределения $\varphi_{k}$ при умеренных значениях $N$.

Двусторонние оценки величин $\mathbf{P}\left\{x \in S_{k} \mid \varphi_{0}=n\right\}, \mathbf{P}\left\{x \in \Psi_{k} \mid \varphi_{0}=n\right\}$ и первых моментов случайных величин $\varphi_{k}, \zeta_{k}$ содержатся в следующей теореме.

Теорема 1. Пусть $F_{1}, F_{2}, \ldots$ - независимые равновероятные отображения множества $\mathcal{N}=\{1, \ldots, N\}$ в себя, $S_{0} \subseteq \mathcal{N},\left|S_{0}\right|=n, S_{k}=F_{k}\left(\ldots\left(F_{1}\left(S_{0}\right)\right) \ldots\right), k \geqslant 1$. Для любого элемента $x \in \mathcal{N}$, не зависящего от отображений $F_{1}, F_{2}, \ldots$, при любых $1 \leqslant k, n \leqslant N$ справедливы неравенства

$$
\begin{gathered}
\frac{n}{N}-C_{n}^{2} \frac{k}{N^{2}} \leqslant \mathbf{P}\left\{x \in S_{k} \mid \varphi_{0}=n\right\}<\frac{n}{N}-C_{n \frac{k}{N^{2}}+\frac{n^{3} k^{2}}{4 N^{3}}}^{2} \\
\frac{n t}{N}-C_{t+1}^{2} \frac{3 n^{2}}{2 N^{2}}<\mathbf{P}\left\{x \in \Psi_{t} \mid \varphi_{0}=n\right\}<\frac{n t}{N}-C_{n}^{2} C_{t+1}^{2} \frac{1}{N^{2}}+\frac{n^{3}(t+1)^{3}}{12 N^{3}} .
\end{gathered}
$$


Справедливы также следующие оценки:

$$
\begin{gathered}
n-C_{n}^{2} \frac{k}{N} \leqslant \mathbf{M}\left\{\varphi_{k} \mid \varphi_{0}=n\right\}<n-C_{n}^{2} \frac{k}{N}+\frac{n^{3} k^{2}}{4 N^{2}} \\
n t-C_{t+1}^{2} \frac{3 n^{2}}{2 N}<\mathbf{M}\left\{\zeta_{t} \mid \varphi_{0}=n\right\}<n t-C_{n}^{2} C_{t+1}^{2} \frac{1}{N}+\frac{n^{3}(t+1)^{3}}{12 N^{2}} \\
\mathbf{D}\left\{\varphi_{k} \mid \varphi_{0}=n\right\}<\frac{k n^{3}}{N}\left(1+\frac{(n+2) k}{4 n N}\right) .
\end{gathered}
$$

Доказательство. Будем использовать обозначение $F_{k \ldots 1}(x)$ для $F_{k}\left(\ldots\left(F_{1}(x)\right) \ldots\right)$. Из равновероятности отображений $F_{j}, j \geqslant 1$, следует, что величина $\mathbf{P}\left\{x \in S_{k}|| S_{0} \mid=n\right\}$ не зависит от $x \in \mathcal{N}$ как при фиксированном $x$, так и при случайном $x$, принимающем значения в $\mathcal{N}$ и не зависящем от $F_{1}, F_{2}, \ldots$ Поэтому неравенства (4) будем доказывать для $x=1$. Так как

$$
\left\{1 \in S_{k}\right\}=\bigcup_{x \in S_{0}}\left\{F_{k \ldots 1}(x)=1\right\}
$$

то в силу неравенств Бонферрони

$$
\begin{gathered}
\sum_{x \in S_{0}} \mathbf{P}\left\{F_{k \ldots 1}(x)=1\right\}-\sum_{\substack{x, y \in S_{0} \\
x<y}} \mathbf{P}\left\{F_{k \ldots 1}(x)=F_{k \ldots 1}(y)=1\right\} \leqslant \\
\leqslant \mathbf{P}\left\{1 \in S_{k} \mid \varphi_{0}=n\right\} \leqslant \\
\leqslant \sum_{x \in S_{0}} \mathbf{P}\left\{F_{k \ldots 1}(x)=1\right\}-\sum_{\substack{x, y \in S_{0} \\
x<y}} \mathbf{P}\left\{F_{k \ldots 1}(x)=F_{k \ldots 1}(y)=1\right\}+ \\
+\sum_{\substack{x, y, z \in S_{0} \\
x<y<z}} \mathbf{P}\left\{F_{k \ldots 1}(x)=F_{k \ldots 1}(y)=F_{k \ldots 1}(z)=1\right\} .
\end{gathered}
$$

Очевидно,

$$
\mathbf{P}\left\{F_{k \ldots 1}(x)=1\right\}=\frac{1}{N} \text { для любого } x \in \mathcal{N} .
$$

Далее, при любых $x, y \in \mathcal{N}, x \neq y$,

$$
\begin{gathered}
\mathbf{P}\left\{F_{k \ldots 1}(x)=F_{k \ldots 1}(y)=1\right\}= \\
=\mathbf{P}\left\{F_{k \ldots 1}(x)=1\right\} \mathbf{P}\left\{F_{k \ldots 1}(y)=F_{k \ldots 1}(x) \mid F_{k \ldots 1}(x)=1\right\}= \\
=\frac{1}{N} \mathbf{P}\left\{\bigcup_{j=1}^{k}\left[\min \left\{i: F_{i \ldots 1}(y)=F_{i \ldots 1}(x)\right\}=j\right]\right\},
\end{gathered}
$$

поскольку в силу равновероятности отображений $F_{j}$ условная вероятность не зависит от значения $F_{k \ldots 1}(x)$. События в правой части $(9)$ несовместны, и в силу независимости и равновероятности отображений $F_{1}, F_{2}, \ldots$ при любом $j=1, \ldots, k$

$$
\mathbf{P}\left\{\left\{\min \left\{i: F_{k \ldots 1}(y)=F_{k \ldots 1}(x)\right\}=j\right\}\right\}=\frac{1}{N}\left(1-\frac{1}{N}\right)^{j-1},
$$

T. e.

$$
\begin{gathered}
\mathbf{P}\left\{F_{k \ldots 1}(x)=F_{k \ldots 1}(y)=1\right\}= \\
=\frac{1}{N} \mathbf{P}\left\{\bigcup_{j=1}^{k}\left\{\left[\min \left\{i: F_{i \ldots 1}(y)=F_{i \ldots 1}(x)\right\}=j\right]\right\}=\right. \\
=\frac{1}{N} \sum_{j=1}^{k} \frac{1}{N}\left(1-\frac{1}{N}\right)^{j-1}=\frac{1}{N}\left(1-\left(1-\frac{1}{N}\right)^{k}\right) \in\left[\frac{k}{N}-C_{k}^{2} \frac{1}{N^{2}}, \frac{k}{N}\right] .
\end{gathered}
$$


Аналогично для любых попарно различных $x, y, z \in \mathcal{N}$

$$
\begin{gathered}
\mathbf{P}\left\{F_{k \ldots 1}(x)=F_{k \ldots 1}(y)=F_{k \ldots 1}(z)=1\right\}= \\
=\mathbf{P}\left\{F_{k \ldots 1}(x)=1\right\} \mathbf{P}\left\{F_{k \ldots 1}(z)=F_{k \ldots 1}(y)=F_{k \ldots 1}(x) \mid F_{k \ldots 1}(x)=1\right\}= \\
=\frac{1}{N} \mathbf{P}\left\{\bigcup _ { j , m = 1 } ^ { k } \left[\min \left\{i: F_{i \ldots 1}(y)=F_{i \ldots 1}(x)\right\}=j,\right.\right. \\
\left.\left.\min \left\{r: F_{r \ldots 1}(z) \in\left\{F_{r \ldots 1}(x), F_{r \ldots 1}(y)\right\}\right\}=m\right]\right\} .
\end{gathered}
$$

При разных парах $(j, m)$ события в правой части несовместны и

$$
\begin{gathered}
\mathbf{P}\left\{\min \left\{i: F_{i \ldots 1}(y)=F_{i \ldots 1}(x)\right\}=j,\right. \\
\left.\min \left\{r: F_{r \ldots 1}(z) \in\left\{F_{r \ldots 1}(x), F_{r \ldots 1}(y)\right\}\right\}=m\right\}= \\
= \begin{cases}\frac{1}{N}\left(1-\frac{1}{N}\right)^{j-1} \frac{2}{N}\left(1-\frac{2}{N}\right)^{m-1}, & m<j, \\
\frac{1}{N}\left(1-\frac{1}{N}\right)^{j-1} \frac{1}{N}\left(1-\frac{2}{N}\right)^{j-1}\left(1-\frac{1}{N}\right)^{m-j}, \quad m \geqslant j .\end{cases}
\end{gathered}
$$

Так как

$$
\begin{aligned}
& \sum_{j=2}^{k} \sum_{m=1}^{j-1} \frac{1}{N}\left(1-\frac{1}{N}\right)^{j-1} \frac{2}{N}\left(1-\frac{2}{N}\right)^{m-1}<\frac{2}{N^{2}} \sum_{j=1}^{k-1} j=\frac{k(k-1)}{N^{2}} \\
& \sum_{m=1}^{k} \sum_{j=1}^{m} \frac{1}{N}\left(1-\frac{1}{N}\right)^{m-1} \frac{1}{N}\left(1-\frac{2}{N}\right)^{j-1}<\frac{1}{N^{2}} \sum_{m=1}^{k} m=\frac{k(k+1)}{2 N^{2}}
\end{aligned}
$$

Tо

$$
\mathbf{P}\left\{F_{k \ldots 1}(x)=F_{k \ldots 1}(y)=F_{k \ldots 1}(z)=1\right\}<\frac{k(k-1)}{N^{2}}+\frac{k(k+1)}{2 N^{2}}<\frac{3 k^{2}}{2 N^{3}} .
$$

Из (7), (8), (10), (11) следует, что

$$
\begin{gathered}
\mathbf{P}\left\{1 \in S_{k} \mid \varphi_{0}=n\right\} \geqslant \frac{n}{N}-C_{n}^{2} \frac{k}{N^{2}}, \\
\mathbf{P}\left\{1 \in S_{k} \mid \varphi_{0}=n\right\} \leqslant \frac{n}{N}-C_{n}^{2} \frac{1}{N}\left(1-\left(1-\frac{1}{N}\right)^{k}\right)+C_{n}^{3} \frac{3 k^{2}}{2 N^{3}} \leqslant \\
\leqslant \frac{n}{N}-C_{n}^{2} \frac{k}{N^{2}}+C_{n}^{2} C_{k}^{2} \frac{1}{N^{3}}+C_{n}^{3} \frac{3 k^{2}}{2 N^{3}}<\frac{n}{N}-C_{n}^{2} \frac{k}{N^{2}}+\frac{n^{3} k^{2}}{4 N^{3}} .
\end{gathered}
$$

Тем самым первое неравенство в (4) доказано.

Доказательство второго неравенства тоже проводится с помощью неравенств Бонферрони

$$
\begin{gathered}
\sum_{k=1}^{t} \mathbf{P}\left\{1 \in S_{k} \mid \varphi_{0}=n\right\}-\sum_{1 \leqslant k<m \leqslant t} \mathbf{P}\left\{1 \in S_{k}, 1 \in S_{m} \mid \varphi_{0}=n\right\} \leqslant \\
\leqslant \mathbf{P}\left\{1 \in \Psi_{t}=\bigcup_{k=1}^{t} S_{k} \mid \varphi_{0}=n\right\}=\mathbf{P}\left\{\bigcup_{k=1}^{t}\left\{1 \in S_{k}\right\} \mid \varphi_{0}=n\right\} \leqslant \\
\leqslant \sum_{k=1}^{t} \mathbf{P}\left\{1 \in S_{k}|| S_{0} \mid=n\right\} .
\end{gathered}
$$


Используя первые неравенства в (4), находим:

$$
\begin{gathered}
\frac{n t}{N}-C_{n}^{2} C_{t+1}^{2} \frac{1}{N^{2}}=\sum_{k=1}^{t}\left(\frac{n}{N}-C_{n}^{2} \frac{k}{N^{2}}\right) \leqslant \sum_{k=1}^{t} \mathbf{P}\left\{1 \in S_{k} \mid \varphi_{0}=n\right\} \leqslant \\
\leqslant \sum_{k=1}^{t}\left(\frac{n}{N}-C_{n}^{2} \frac{k}{N^{2}}+\frac{n^{3} k^{2}}{4 N^{3}}\right)<\frac{n t}{N}-C_{n}^{2} C_{t+1}^{2} \frac{1}{N^{2}}+\frac{n^{3}(t+1)^{3}}{12 N^{3}} .
\end{gathered}
$$

Далее, с учетом того, что при условии вида $\varphi_{k}=i, \varphi_{m}=j$ множества $S_{k}$ и $S_{m}$ независимы, не зависят от предыстории и что последовательность $\varphi_{r}, r \geqslant 0$, образует цепь Маркова с невозрастающими траекториями, находим, что при $1 \leqslant k<m$

$$
\begin{gathered}
\mathbf{P}\left\{1 \in S_{k}, 1 \in S_{m} \mid \varphi_{0}=n\right\}= \\
=\sum_{n \geqslant i \geqslant j \geqslant 1} \mathbf{P}\left\{1 \in S_{k}, 1 \in S_{m}, \varphi_{k}=i, \varphi_{m}=j \mid \varphi_{0}=n\right\}= \\
=\sum_{i=1}^{n} \mathbf{P}\left\{\varphi_{k}=i \mid \varphi_{0}=n\right\} \frac{i}{N} \sum_{j=1}^{i} \mathbf{P}\left\{\varphi_{m}=j \mid \varphi_{k}=i\right\} \frac{j}{N} \leqslant \\
\leqslant \sum_{i=1}^{n} \mathbf{P}\left\{\varphi_{k}=i \mid \varphi_{0}=n\right\} \frac{i}{N} \frac{i}{N} \leqslant \frac{1}{N^{2}} \mathbf{E}\left\{\varphi_{k}^{2} \mid \varphi_{0}=n\right\} \leqslant \frac{n^{2}}{N^{2}} .
\end{gathered}
$$

Из (12), (13), (14) следует второе неравенство в (4):

$$
\begin{gathered}
\frac{n t}{N}-C_{t+1}^{2} \frac{3 n^{2}}{2 N^{2}}<\frac{n t}{N}-C_{n}^{2} C_{t+1}^{2} \frac{1}{N^{2}}-C_{t}^{2} \frac{n^{2}}{N^{2}} \leqslant \mathbf{P}\left\{1 \in \Psi_{t} \mid \varphi_{0}=n\right\}< \\
<\frac{n t}{N}-C_{n}^{2} C_{t+1}^{2} \frac{1}{N^{2}}+\frac{n^{3}(t+1)^{3}}{12 N^{3}} .
\end{gathered}
$$

Неравенства для математических ожиданий непосредственно следуют из (4), так как

$$
\varphi_{k}=\sum_{j=1}^{N} \mathbb{I}\left\{j \in S_{k}\right\}, \quad \zeta_{t}=\sum_{j=1}^{N} \mathbb{I}\left\{j \in \Psi_{t}\right\} .
$$

Для оценки $\mathbf{D}\left\{\varphi_{k} \mid \varphi_{0}=n\right\}$ используем (15), а также независимость и равновероятность отображений $F_{k}, k \geqslant 1$ :

$$
\begin{gathered}
\mathbf{D}\left\{\varphi_{k} \mid \varphi_{0}=n\right\}=\mathbf{M}\left\{\varphi_{k}^{2} \mid \varphi_{0}=n\right\}-\left(\mathbf{M}\left\{\varphi_{k} \mid \varphi_{0}=n\right\}\right)^{2}= \\
=\mathbf{M}\left\{\sum_{i, j=1}^{N} \mathbb{I}\left\{i, j \in S_{k}\right\} \mid \varphi_{0}=n\right\}-\left(\mathbf{M}\left\{\varphi_{k} \mid \varphi_{0}=n\right\}\right)^{2}= \\
=\mathbf{M}\left\{\varphi_{k} \mid \varphi_{0}=n\right\}+N(N-1) \mathbf{P}\left\{1,2 \in S_{k} \mid \varphi_{0}=n\right\}-\left(\mathbf{M}\left\{\varphi_{k} \mid \varphi_{0}=n\right\}\right)^{2} .
\end{gathered}
$$

Оценки для $\mathbf{M}\left\{\varphi_{k} \mid \varphi_{0}=n\right\}$ уже получены. Далее, в силу равновероятности и независимости отображений $F_{k}, k \geqslant 1$,

$$
\begin{gathered}
\mathbf{P}\left\{1,2 \in S_{k} \mid \varphi_{0}=n\right\}=\mathbf{P}\left\{\bigcup_{x, y=1}^{n}\left\{F_{k \ldots 1}(x)=1, F_{k \ldots 1}(y)=2\right\}\right\} \leqslant \\
\leqslant \sum_{x, y=1}^{n} \mathbf{P}\left\{F_{t \ldots 1}(x) \neq F_{t \ldots 1}(y)(1 \leqslant t<k), F_{k \ldots 1}(x)=1, F_{k \ldots 1}(y)=2\right\}= \\
=n(n-1)\left(1-\frac{1}{N}\right)^{k-1} \frac{1}{N^{2}} .
\end{gathered}
$$


Из (5), (16), (17) и неравенства $\left(1-\frac{1}{N}\right)^{k} \leqslant 1-\frac{k}{N}+C_{k}^{2} \frac{1}{N^{2}}$ следует, что

$$
\begin{gathered}
\mathbf{D}\left\{\varphi_{k} \mid \varphi_{0}=n\right\}< \\
<n-C_{n}^{2} \frac{k}{N}+\frac{n^{3} k^{2}}{4 N^{2}}+\frac{(N-1) n(n-1)}{N}\left(1-\frac{1}{N}\right)^{k-1}-\left(n-C_{n}^{2} \frac{k}{N}\right)^{2}= \\
=\frac{n^{3} k^{2}}{4 N^{2}}+n(n-1)\left(\left(1-\frac{1}{N}\right)^{k}-\left(1-\frac{k(n-1)}{2 N}\right)\left(1-\frac{k n}{2 N}\right)\right) \leqslant \\
\leqslant \frac{n^{3} k^{2}}{4 N^{2}}+n(n-1)\left(\left(1-\frac{k}{N}+\frac{k(k-1)}{2 N^{2}}\right)-\left(1-\frac{k(2 n-1)}{2 N}+\frac{k n(n-1)}{4 N^{2}}\right)\right)= \\
=\frac{n^{3} k^{2}}{4 N^{2}}+n(n-1)\left(\frac{k(2 n-1)-2 k}{2 N}-\frac{k n(n-1)-2 k(k-1)}{4 N^{2}}\right)<\frac{k n^{3}}{N}\left(1+\frac{(n+2) k}{4 n N}\right) .
\end{gathered}
$$

Тем самым неравенство (6) и теорема 1 доказаны.

Неравенства теоремы можно уточнять, если использовать больше членов в неравенствах Бонферрони.

\section{Список литературы}

1. Гульден Я., Джексон Д., Перечислительная комбинаторика, М. : Наука. Физматлит, 1990, $503 \mathrm{c}$.

2. Зубков А. М., Шибанов О. К., "Время до объединения всех частиц при равновероятных размещениях по последовательности слоев ячеек", Матем. заметки, 85:3 (2009), $373-381$.

3. Колчин В. Ф., Случайнъе отображения, М. : Наука, 1984, 208 с.

4. Колчин В. Ф., Севастьянов Б. А., Чистяков В. П., Случайные размещения, М. : Наука, 1976, $224 \mathrm{c}$.

5. Степанов В. Е., “О распределении числа вершин в слоях случайного дерева”, Теория вероятн. и примен., 14:1 (1969), 64-77.

6. Dalal A., Schmutz E. "Compositions of random functions on a finite set", Electr. J. Comb., 9:R26 (2002).

7. Flajolet P., Odlyzko A. M., "Random mapping statistics", Advances in Cryptology, Proc. Eurocrypt'89, Lect. Notes Comput. Sci., 434, 1990, 329-354.

8. Goh W. M. Y., Hitczenko P., Schmutz E., "Iterating random functions on a finite set", 2014, 7 pp., arXiv:math/0207276v2.

9. Harris B., "Probability distributions related to random mappings", Ann. Math. Statist., 31:2 (1960), 1045-1062.

10. Hellman M.E., “A cryptanalytic time-memory trade-off”, IEEE Trans. Inf. Theory, 1980, 401-406.

11. Hong J., Ma D., "Success probability of the Hellman trade-off", Inf. Process. Lett., 109:7 (2009), 347-351.

12. Kingman J. F. C., "The coalescent", Stoch.Proc. Appl., 13 (1982), 235-248.

13. Kusuda K., Matsumoto T., "Optimization of time-memory trade-off cryptanalysis and its application to DES", IEICE Trans. on Fundamentals, 1:E-79A (1996), 35-48.

14. McSweeney J.K., Pittel B.G., "Expected coalescence time for a nonuniform allocation process", Adv. Appl. Probab., 40:4 (2008), 1002-1032.

15. Oechslin P., "Making a faster cryptanalytic time-memory trade-off", Lect. Notes Comput. Sci., 2729 (2003), 617-630.

16. Pilshchikov D. V., "Estimation of the characteristics of time-memory-data tradeoff methods via generating functions of the number of particles and the total number of particles in the Galton-Watson process", Математические вопросы криптографии, 5:2 (2014), 103-108.

17. Rubin H., Sitgreaves R., Probability distributions related to random transformations of a finite set, Tech. report. №19A, Appl. math. and statist. lab., Stanford Univ., 1954. 